\title{
COMPORTAMIENTO SÍSMICO DE EDIFICIOS DE ACERO CON MARCOS PERIMETRALES RESISTENTES A MOMENTO
}

\author{
Alfredo Reyes Salazar ${ }^{(1)}$, Juan Bojórquez Mora ${ }^{(2)}$, Edén Bojórquez Mora ${ }^{(1)}$, \\ Juan I. Velázquez Dimas ${ }^{(1)}$ y Héctor E. Rodríguez Lozoya ${ }^{(I)}$
}

\begin{abstract}
RESUMEN
Varios aspectos relacionados con la idealización estructural de edificios de acero con marcos perimetrales resistentes a momento y marcos interiores de gravedad (MGs) se abordan en esta investigación. El estudio numérico indica que la contribución de los MGs a la resistencia lateral puede ser significativa y que modelar los edificios como marcos planos puede resultar en elementos mecánicos, cortantes y desplazamientos de entrepiso más grandes que cuando se modelan en forma tridimensional, por lo que su diseño puede ser conservador. También se observa que el cortante de entrepiso promedio generalmente se incrementa cuando se considera la rigidez de las conexiones de los MGs. Los desplazamientos de entrepiso promedio son similares para los modelos con conexiones articuladas y semi-rígidas. Los resultados también indican que los momentos que las conexiones pueden transmitir son cercanos al $30 \%$ de los momentos plásticos de las vigas a las que conectan. La diferencia entre los resultados se debe principalmente a los elementos que contribuyen a la resistencia y rigidez, la disipación de energía, y las características dinámicas de cada representación estructural. Se concluye que, si se usa la estructuración antes mencionada, el modelo tridimensional debe ser usado, que los MGs deberán considerarse como parte del sistema lateral resistente y que la rigidez de las conexiones deberá incluirse en el diseño de los MGs.
\end{abstract}

\begin{abstract}
Several issues regarding the structural idealization of steel buildings with perimeter moment resisting steel frames and interior gravity frames (IGFs) are addressed in this paper. The numerical study indicates that the contribution of IGFs to the lateral structural resistance may be significant and that modeling the buildings as plane frames may result in larger resultant stresses, interstory shears and displacements implying that the design may be conservative. It is also observed that the average interstory shear generally increases when the connections stiffness of the IGFs is taken into account. The average interstory displacements are similar for the models with pinned and semirigid connections. The results also indicate that the maximum moments at the connections may be up to $30 \%$ of the plastic moments of the beams they are connecting to. In general, the differences observed in the behavior of each structural representation are mainly due to a) the elements that contribute to strength and stiffness, b) the energy dissipation characteristics, and c) the dynamics characteristics of each structural representation. It is concluded that, if the above-mentioned structural system is used, the three-dimensional model should be used in seismic analysis, that the IGFs should be considered as part of the lateral resistance system, and that the stiffness of the connections should be included in the design of the IGFs.
\end{abstract}

\footnotetext{
(1) Profesores, Facultad de Ingeniería, Universidad Autónoma de Sinaloa, Calzada de las Américas y Boulevard Universitarios S/N, Ciudad Universitaria, Culiacán, Sinaloa, México, CP 80040, reyes@uas.uasnet.mx, eden_bmseg@hotmail.com, juanv@uas.uasnet.mx, rolohe@uas.uasnet.mx

${ }^{(2)}$ Estudiantes, Facultad de Ingeniería, Universidad Autónoma de Sinaloa, Calzada de las Américas y Boulevard Universitarios S/N, Ciudad Universitaria, Culiacán, Sinaloa, México, CP 80040,jbm_squall_cloud_@hotmail.com.
} 


\section{INTRODUCCIÓN}

El objetivo central de las previsiones de diseño sísmico especificados en los códigos es proveer a las estructuras con la capacidad suficiente para resistir terremotos severos sin llegar al colapso, aunque con algún daño estructural. Diferentes sistemas estructurales y materiales se usan para lograr este fin. Para el caso de edificios de acero, entre los diferentes sistemas estructurales, los marcos resistentes a momento (MRMs) a base de secciones tipo $W$ han sido muy usados porque permiten la optimización de los espacios y por su gran capacidad de ductilidad. Sin embargo, estos sistemas estructurales, han cambiado significativamente con el paso de los años, particularmente en Estados Unidos. De mediados de los 60's a mediados de los 70's, la mayoría de las conexiones en edificios de acero fueron conexiones resistentes a momento (CRMs). En los últimos años, el uso de CRMs fue tremendamente reducido debido a su costo excesivo y para eliminar conexiones rígidas respecto al eje-débil. Después del Terremoto de Northridge de 1994, la Agencia Federal de Emergencias de Estados Unidos, FEMA por sus siglas en Inglés (FEMA, 2000), sugirió el uso de un sistema estructural con MRMs en la periferia y marcos de gravedad (MGs) en el interior y propusieron algunos modelos. La ventaja principal de tal arreglo estructural radica en la simplicidad del análisis puesto que permite realizar el diseño de los MRMs bajo la acción únicamente de las cargas sísmicas totales y el diseño de los MGs sometidos a la acción de las cargas de gravedad. Aquí se considera que cada MRMs se comporta bidimensionalmente dentro de una estructura tridimensional. Otra ventaja importante es la eliminación de la flexión respecto al eje débil. La desventaja principal consiste en los errores introducidos en la estimación de la respuesta por la simplificación. El comportamiento del sistema estructural en cuestión sometido a la acción de cargas sísmicas es aún una "pregunta abierta" y necesita ser estudiado. En parte ese es el objetivo principal de esta investigación.

Como se comentó anteriormente, con el objeto de simplificar el proceso de análisis y diseño sísmico los edificios tridimensionales se modelan como marcos planos. En esta idealización se ignora la presencia de los MGs. Debido a la acción de la rigidez de la losa, sin embargo, estos MGs sufrirán una deformación lateral similar a la de los MRMs y en consecuencia la contribución de las columnas de estos marcos a la resistencia lateral podría ser significativa, particularmente para aquellos edificios con relativamente pocas CRMs. Además, la modelación de los edificios como marcos planos puede no representar el comportamiento real de la estructura puesto que, además de no considerar la participación de algunos elementos estructurales, las propiedades dinámicas de este modelo pueden ser diferentes de las del modelo tridimensional.

Otra simplificación hecha en el diseño de edificios de acero con MRMs perimetrales y MGs en el interior es en la rigidez de la conexión viga-columna de los MGs. Los análisis y diseños convencionales de marcos de acero se basan en la suposición de que las conexiones viga-columna son perfectamente rígidas (PR) o perfectamente articuladas (PA). En el sistema estructural anteriormente discutido, la conexión viga-columna de los MGs se asume PA, aunque en la práctica se usan conexiones de cortante. A pesar de estas clasificaciones, casi todas las conexiones de acero usadas en marcos reales son esencialmente semi-rígidas (SR) con diferentes niveles de rigidez. Se reconoce en la profesión tanto teórica como experimentalmente que las conexiones tienen una respuesta semi-rígida no lineal aun si la amplitud de la carga aplicada es muy pequeña (Reyes-Salazar y Haldar 2000). La consideración de conexiones PR y PA es únicamente una suposición para simplificar los cálculos y representa una debilidad en los procedimientos analíticos actuales. Estas simplificaciones pueden resultar en valores erróneos de los elementos mecánicos, porque en realidad las conexiones PR poseen algo de flexibilidad y las conexiones PA poseen algo de rigidez. Hay evidencias de que las conexiones de cortante pueden transmitir hasta el $30 \%$ de la capacidad de momento plástico (FEMA 2000) de las vigas a las que están conectadas. La contribución de estas conexiones a la resistencia y rigidez estructural puede ser aun más importante si se considera la acción compuesta de la losa (Reyes- Salazar y Haldar, 1999; Liu y AstanehAs1, 2000). 


\section{OBJETIVOS}

En este artículo se estudian varios aspectos relacionados con la idealización estructural de edificios de acero con marcos perimetrales resistentes a momento y marcos interiores de gravedad. Específicamente se estudia: 1) la contribución de los MGs a la resistencia lateral; 2) la precisión de modelar los edificios tridimensionales como marcos planos para fines de análisis y diseño sísmico; 3) la diferencia entre las respuestas sísmicas de edificios con conexiones PA y las correspondientes respuestas de los edificios con conexiones SR y 4) la magnitud de los momentos desarrollados en las conexiones SR. Las respuestas se estiman en términos de parámetros globales (cortante basal y desplazamientos de entrepiso) y locales (elementos mecánicos en miembros individuales). Algunos modelos propuestos por la Agencia Federal de Emergencias de Estados Unidos (FEMA, 2000) se usan para este propósito. Los modelos se analizan en el dominio del tiempo bajo la acción de 20 registros sísmicos. Dichos registros sísmicos fueron obtenidos de conjuntos de datos del Programa Nacional de Movimientos Fuertes (NSMP por sus siglas en inglés) del Banco de Datos Geológicos de Estados Unidos (USGS por sus siglas en inglés)y fueron seleccionados para representar las características de terremotos fuertes.

\section{FORMULACIÓN MATEMÁTICA.}

Para lograr los objetivos del estudio, la respuesta sísmica de los modelos usados se evalúa de la manera más realista posible usando un procedimiento de análisis de elemento finito basado en hipótesis de esfuerzos desarrollado por los autores y un equipo de investigadores (Reyes-Salazar 1997, Gao and Haldar 1995). El procedimiento estima la respuesta sísmica no lineal en el dominio del tiempo considerando las no linealidades geométrica, del material, y la introducida por las conexiones SR. Una forma explícita de la matriz de rigidez tangente puede obtenerse sin requerir integración numérica. Configuraciones de deformaciones grandes pueden ser descritas usando pocos elementos sin perder exactitud, y las no linealidades pueden incorporarse sin perder su simplicidad básica. El procedimiento arroja resultados muy precisos y es muy eficiente comparado con la aproximación basada en desplazamientos. El procedimiento y el algoritmo han sido verificados utilizando resultados disponibles tanto teóricos como experimentales (Reyes-Salazar y Haldar 2001a, Reyes-Salazar y Haldar 2001b, Gao y Haldar 1995).

\section{EL MODELO DE RICHARD}

Las resultantes de esfuerzos (fuerzas axiales y cortantes, y momentos torsionantes y flexionantes) se transmiten entre los diferentes elementos de una estructura a través de sus conexiones. Como se discutió previamente casi todas las conexiones usadas en marcos de acero son esencialmente SR con diferentes niveles de rigidez. El momento flexionante en la conexión y su correspondiente rotación relativa, denotados como curva momento-rotación $(M-\theta)$, se utilizan generalmente para representar el comportamiento flexible de las conexiones.

Varias alternativas se encuentran disponibles en la literatura para definir las curvas $M-\theta$ (ReyesSalazar 1997): El Modelo Lineal por Segmentos, El Modelo Polinomial, El Modelo Exponencial, y El Modelo de Richard (Richard 1993), entre otros. El modelo de Richard se usa en este estudio. Dicho modelo fue desarrollado utilizando información experimental y se aplica a una amplia variedad de conexiones. De acuerdo a este modelo la curva $M$ - $\theta$ está dada por 


$$
M=\frac{\left(k-k_{p}\right) \theta}{\left(1+\left|\frac{\left(k-k_{p}\right) \theta}{M_{o}}\right|^{N}\right)^{\frac{1}{N}}}+k_{p} \theta
$$

donde $k$ es la rigidez inicial o elástica, $k_{p}$ es la rigidez plástica, $M_{0}$ es el momento de referencia y $N$ es el parámetro de forma de la curva. La definición física de estos parámetros se muestra en la figura 1.

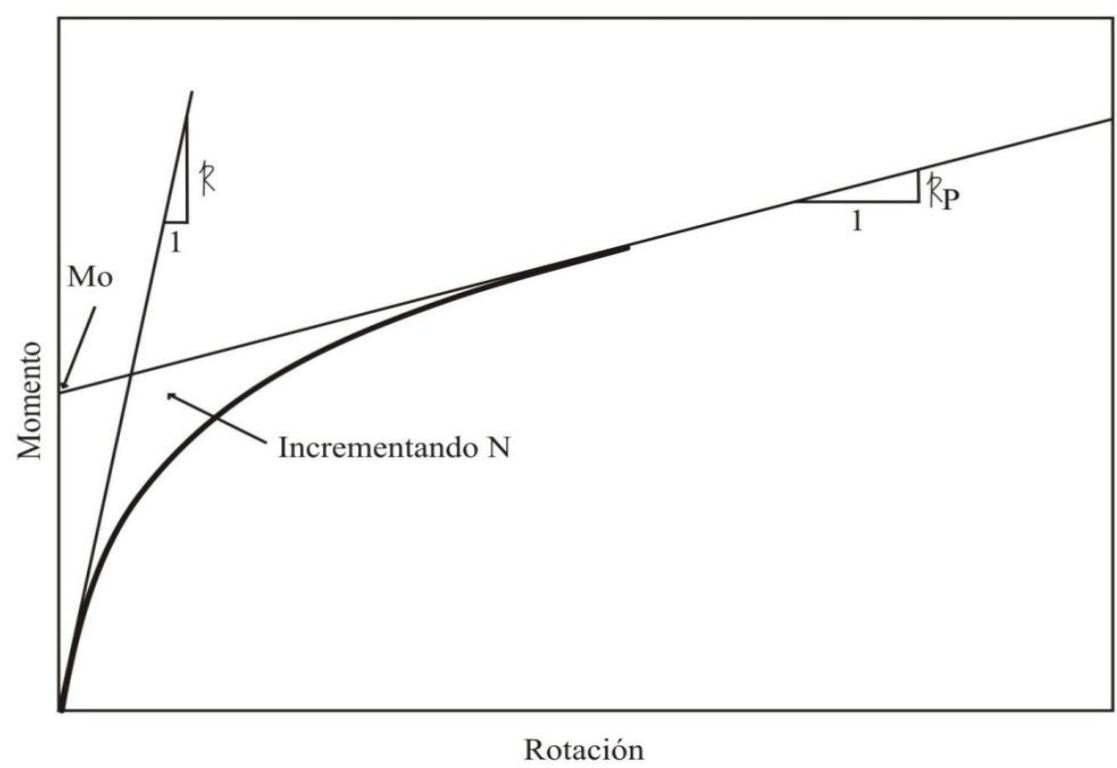

Figura 1. Parámetros del Modelo de Richard

La ecuación 1 representa la etapa donde la carga en la conexión se incrementa monotónicamente. En un análisis sísmico típico, para un instante de tiempo dado, se espera que algunas conexiones estén en el proceso de carga mientras que otras pueden estar en el proceso de descarga o recarga. Estudios relacionados con el comportamiento de carga, descarga y recarga en las conexiones SR, tanto experimentales como teóricos son poco comunes. Sin embargo, la consideración de dicho comportamiento en el análisis sísmico es esencial. Este tópico ha sido abordado por Colson (1991) y El-Salti (1992). En estos estudios, el Modelo de Richard y la Regla de Masing se usan para representar las secciones de descarga y recarga de la curva $M-\theta$ (Reyes Salazar y otros, 2001) y se adoptan en esta investigación.

\section{MODELOS ESTRUCTURALES}

Como parte del proyecto de la SAC, tres prestigiadas firmas de consultorías de Estados Unidos fueron comisionadas por FEMA (2000) para realizar el diseño de varios modelos de edificios de acero con MRMs perimetrales y MGs en el interior. Los modelos son de 3, 9 y 20 niveles y fueron diseñados de acuerdo a los códigos de construcción de las siguientes tres ciudades: Los Ángeles (UBC, 1994), Seattle (UBC, 1994) y Boston (BOCA, 1993). Los modelos de 3 y 9 niveles, representando la zona de Los Ángeles y los diseños pre-Northridge, se usan en esta investigación para estudiar los problemas mencionados anteriormente. Estos modelos se denotan de aquí en adelante como Modelos 1 y 2 y sus periodos fundamentales son 1.03 y 2.34 seg., respectivamente. La elevación de los modelos se da en las 
figuras $2 \mathrm{a}$ y $2 \mathrm{~b}$ y sus plantas en las figuras $3 \mathrm{a}$ y $3 \mathrm{~b}$. En éstas, las líneas continuas representan MRMs y las líneas punteadas MGs. Obsérvese que para el caso del Modelo 1 no hay intercepción de los MRMs por lo que no existe flexión respecto al eje débil. Para el caso del Modelo 2, los marcos perimetrales se interceptan en la esquina; en este caso, sin embargo, las conexiones viga-columna se construyen articuladas para eliminar la flexión respecto al eje débil.

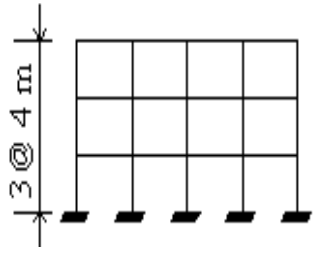

a) Elevación, Modelo 1

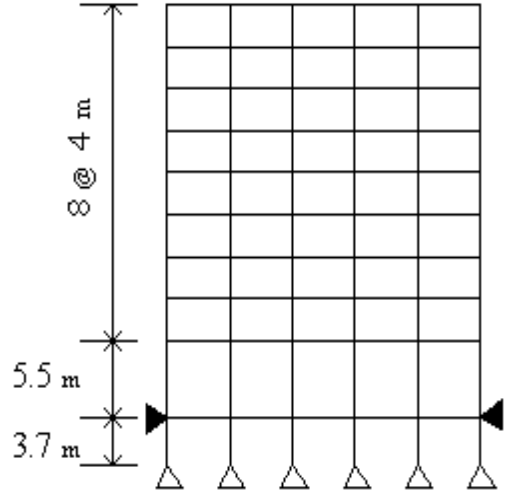

b) Elevación, Modelo 2

Figura 2. Elevación de los Modelos 1 y 2

De las figuras se observa que los marcos son prácticamente simétricos en planta por lo que no se esperan momentos torsionantes significativos. Los elementos particulares considerados en este estudio se muestran es las figuras $4 \mathrm{a}$ y $4 \mathrm{~b}$. Las secciones de las vigas y columnas de los modelos se muestran en la tabla 1. Las columnas de los MRMs del Modelo 1 están empotradas en la base mientras que las del Modelo 2 son articuladas. En todos los marcos las columnas son de acero Grado-50 y las vigas son de acero A36. Para los dos modelos, las columnas de gravedad se consideran articuladas en la base. Todas las columnas en los MRMs se flexionan respecto a su eje fuerte, el eje fuerte de las columnas de los MGs se orienta en la dirección $Y$, como se muestra en la figuras 3a y 3b. Los diseños de los MRMs en las 2 direcciones ortogonales son prácticamente iguales. Información adicional sobre los modelos puede ser obtenida de los reportes del proyecto de la SAC (FEMA, 2000).

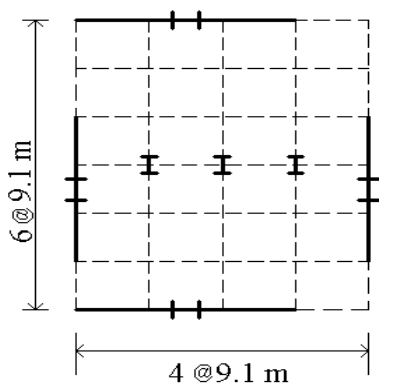

a) Modelo 1

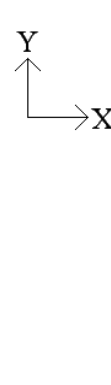

Figura 3. Plantas de los Modelos

Los edificios, en este estudio, se modelan como sistemas de varios grados de libertad (SVGL). Cada columna se representa por un elemento y cada viga de los MRMs se representada por dos elementos, con un nudo en el medio. Se considera que todos los nudos tienen seis grados de libertad. Los modelos se analizan con conexiones PA primero y con conexiones SR después. Un elemento adicional es requerido 
para representar las conexiones SR. Solo es posible considerar conexiones SR para flexión respecto al eje fuerte de las columnas de gravedad. Por lo tanto dichas conexiones están orientadas en dirección $Y$.

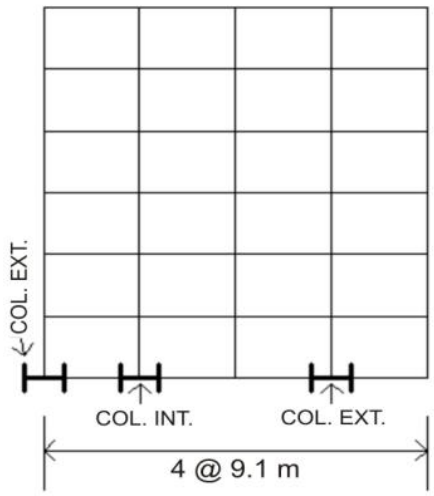

a) Modelo 1
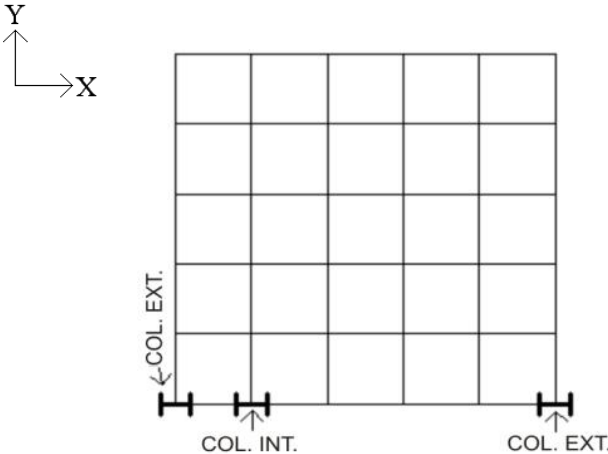

b) Modelo 2

Figura 4. Elementos estudiados

Tabla 1. Secciones de vigas y columnas para los Modelos 1 y 2

\begin{tabular}{|c|c|c|c|c|c|c|c|}
\hline \multirow{3}{*}{ Modelo } & \multicolumn{4}{|c|}{ Marcos resistentes a momento } & \multicolumn{3}{|c|}{ Marcos de gravedad } \\
\hline & \multirow{2}{*}{ Piso } & \multicolumn{2}{|l|}{ Columnas } & \multirow{2}{*}{ Trabes } & \multicolumn{2}{|c|}{ Columnas } & \multirow{2}{*}{ Vigas } \\
\hline & & Exterior & Interior & & Abajo del penthouse & Otros & \\
\hline \multirow{7}{*}{1} & $1 / 2$ & $\mathrm{~W} 14 \times 257$ & $\mathrm{~W} 14 \times 311$ & W33X118 & W14x82 & W14x68 & $\mathrm{W} 18 \times 35$ \\
\hline & $2 / 3$ & $\mathrm{~W} 14 \times 257$ & $\mathrm{~W} 14 \times 312$ & W30X116 & W14x82 & W14x68 & $\mathrm{W} 18 \times 35$ \\
\hline & 3/azotea & $\mathrm{W} 14 \times 257$ & $\mathrm{~W} 14 \times 313$ & W24X68 & W14x82 & W14x68 & $\mathrm{W} 16 \times 26$ \\
\hline & $-1 / 1$ & W14x370 & $\mathrm{W} 14 \times 500$ & W36x160 & $\mathrm{W} 14 \times 211$ & W14x193 & W18x44 \\
\hline & $1 / 2$ & $\mathrm{~W} 14 \times 370$ & $\mathrm{~W} 14 \times 500$ & $\mathrm{~W} 36 \times 160$ & $\mathrm{~W} 14 \times 211$ & W14x193 & W18x35 \\
\hline & $2 / 3$ & $\mathrm{~W} 14 \times 370$ & $\mathrm{~W} 14 \times 500, \mathrm{~W} 14 \times 455$ & W36x160 & W14x211,W14x159 & W14x193,W14x145 & W18x35 \\
\hline & $3 / 4$ & W14x370 & $\mathrm{W} 14 \times 455$ & W36x135 & $\mathrm{W} 14 \times 159$ & $\mathrm{~W} 14 \times 145$ & $\mathrm{~W} 18 \times 35$ \\
\hline \multirow[t]{6}{*}{2} & $4 / 5$ & $\mathrm{~W} 14 \times 370, \mathrm{~W} 14 \times 283$ & $\mathrm{~W} 14 \times 455, \mathrm{~W} 14 \times 370$ & W36x135 & W14x159,W14x120 & W14x145,W14x109 & $\mathrm{W} 18 \times 35$ \\
\hline & $5 / 6$ & $\mathrm{~W} 14 \times 283$ & $\mathrm{~W} 14 \times 370$ & W36x135 & $\mathrm{W} 14 \times 120$ & W14x109 & W18x35 \\
\hline & $6 / 7$ & $\mathrm{~W} 14 \times 283, \mathrm{~W} 14 \times 257$ & W14x370,W14x283 & W36x135 & W14x120,W14x90 & W14x109,W14x82 & $\mathrm{W} 18 \times 35$ \\
\hline & $7 / 8$ & $\mathrm{~W} 14 \times 257$ & $\mathrm{~W} 14 \times 283$ & W30x99 & W14x90 & W14x82 & W18x35 \\
\hline & $8 / 9$ & $\mathrm{~W} 14 \times 257, \mathrm{~W} 14 \times 233$ & W14x283,W14x257 & W27x84 & W14x90,W14x61 & W14x82,W14x48 & $\mathrm{W} 18 \times 35$ \\
\hline & 9/azo & $\mathrm{W} 14 \times 233$ & $\mathrm{~W} 14 \times 257$ & W24x68 & W14x61 & W14x 48 & $\mathrm{~W} 16 \times 26$ \\
\hline
\end{tabular}

\section{ACCIONES SÍSMICAS}

La respuesta dinámica de una estructura sometida a la acción de diferentes registros sísmicos, aún cuando se normalicen con respecto a su máxima aceleración, serán en general diferentes para cada registro, reflejando su diferente contenido de frecuencias. Así que la evaluación de la respuesta estructural usando un solo registro sísmico puede no representar el comportamiento real. Para estudiar los problemas mencionados apropiadamente, los modelos se excitan por veinte terremotos en el dominio del tiempo, registrados en diferentes estaciones. Los criterios principales para la selección de los terremotos fueron: a) la representatividad de éstos de registros en suelos firmes e intermedios y b) aceleración mínima de $0.20 \mathrm{~g}$ durante un tiempo de al menos 20 segundos. Dichos terremotos se dan en la tabla 2, en donde los símbolos $D E, M$ y $A M$, representan la distancia epicentral, magnitud y aceleración máxima, respectivamente. Como se muestra en la tabla, sus periodos predominantes varían desde 0.11 a 1.0. El periodo predominante de estos sismos se define como el valor del periodo correspondiente al pico más 
grande observado en su espectro de respuesta elástico. Dichos registros sísmicos fueron obtenidos de conjuntos de datos del Programa Nacional de Movimientos Fuertes (NSMP por sus siglas en inglés) del Banco de Datos Geológicos de Estados Unidos (USGS por sus siglas en inglés) y fueron seleccionados para representar las características de terremotos fuertes. El amortiguamiento considerado en los análisis es del 5\% del amortiguamiento critico; el mismo que se utiliza en los códigos de diseño.

Tabla 2. Terremotos usados

\begin{tabular}{|c|c|c|c|c|c|c|c|}
\hline No & LUGAR & $\overline{\mathrm{ANNO}}$ & ESTACIÓN & T(seg) & $\overline{\mathrm{DE}}(\mathbf{k m})$ & $\mathbf{M}$ & $\overline{\operatorname{AM}\left(\mathrm{mm} / \mathrm{sec}^{2}\right)}$ \\
\hline 1 & 1317 Mich. México & 1985 & Paraíso & 0.11 & 300 & 8.1 & 800 \\
\hline 2 & 1634 Mammoth Lakes. USA & 1980 & Mammoth H. S. Gym & 0.12 & 19 & 6.5 & 2000 \\
\hline 3 & 1634 Mammoth Lakes USA & 1980 & Convict Creek & 0.19 & 18 & 6.5 & 3000 \\
\hline 4 & 1317 Mich. México & 1985 & Infiernillo $\mathrm{N}-120$ & 0.21 & 67 & 8.1 & 3000 \\
\hline 5 & 1317 Mich. México & 1985 & La Unión & 0.32 & 121 & 8.1 & 1656 \\
\hline 6 & 1733 El Salvador & 2001 & Relaciones Ext. & 0.34 & 96 & 7.8 & 2500 \\
\hline 7 & 1733 El Salvador & 2001 & Relaciones Ext. & 0.41 & 95 & 7.8 & 1500 \\
\hline 8 & 1634 Mammoth Lakes. & 1980 & Long Valley Dam & 0.42 & 13 & 6.5 & 2000 \\
\hline 9 & 2212 Delani Fault, AK & 2000 & K2-02 & 0.45 & 281 & 7.9 & 115 \\
\hline 10 & 0836 Yountville CA & 2000 & Redwood City & 0.46 & 95 & 5.2 & 90 \\
\hline 11 & 0408 Dillon MT & 2005 & MT:Kalispell & 0.51 & 338 & 5.6 & 51 \\
\hline 12 & 1317 Mich. Mexico & 1985 & Villita & 0.53 & 80 & 8.1 & 1225 \\
\hline 13 & 1232 Northrige & 1994 & Hall Valley & 0.54 & 25 & 6.4 & 2500 \\
\hline 14 & 2115 Morgan Hill & 1984 & Hall Valley & 0.61 & 14 & 6.2 & 2000 \\
\hline 15 & 2212 Delani Fault AK & 2002 & K2-04 & 0.62 & 290 & 7.9 & 133 \\
\hline 16 & 0836 Yountville CA & 2000 & Dauville F.S. Ca & 0.63 & 73 & 5.2 & 144 \\
\hline 17 & 0836 Yountville CA & 2000 & Pleasan Hill F.S. 1 & 0.71 & 92 & 5.2 & 74 \\
\hline 18 & 0836 Yountville CA & 2000 & Pleasan Hill F.S. 2 & 0.75 & 58 & 5.2 & 201 \\
\hline 19 & 2212 Delani Fault, AK & 2002 & Valdez City Hall & 0.85 & 272 & 7.9 & 260 \\
\hline 20 & 1715 Park Fiel & 2004 & CA: Hollister City Hall & 1.01 & 147 & 6 & 145 \\
\hline
\end{tabular}

\section{CONTRIBUCIÓN DE LOS MARCOS DE GRAVEDAD}

La contribución de los MGs a la resistencia lateral, en términos de cortantes de entrepiso para los modelos tridimensionales, se estudia en este capítulo. El parámetro $V_{l}$, definido como $V_{I N} / V_{E X}$, se usa para este propósito. Para una dirección y entrepiso dado, $V_{I N}$ representa el cortante de entrepiso resistido por los MGs y $V_{E X}$ el cortante total resistido por los MRMs. Esta razón se estima para ambas direcciones. La componente horizontal con mayor aceleración se aplica en la dirección $X$ mientras que la otra componente se aplica en la dirección $Y$. Esto se denota como $(X, Y)$.

Los modelos con conexiones PA en los MGs se consideran primero. Valores del parámetro $V_{1}$, en porcentaje, se muestran en la figuras 5 a y 5 b para los Modelos 1 y 2, respectivamente, para la dirección $Y$. Se observa que los valores de $V_{l}$ varían significativamente de un modelo a otro y de un piso a otro sin mostrar tendencia alguna. La observación más importante que se puede hacer es que los valores de $V_{l}$, no son despreciables en muchos casos. Valores de hasta $28 \%$ se observan para el Piso 1 del Modelo 1. Gráficas similares a las de la figura 5 también se construyeron para la dirección $X$ pero no se muestran. En general las observaciones realizadas para la dirección $Y$ son válidas para la dirección $X$. 


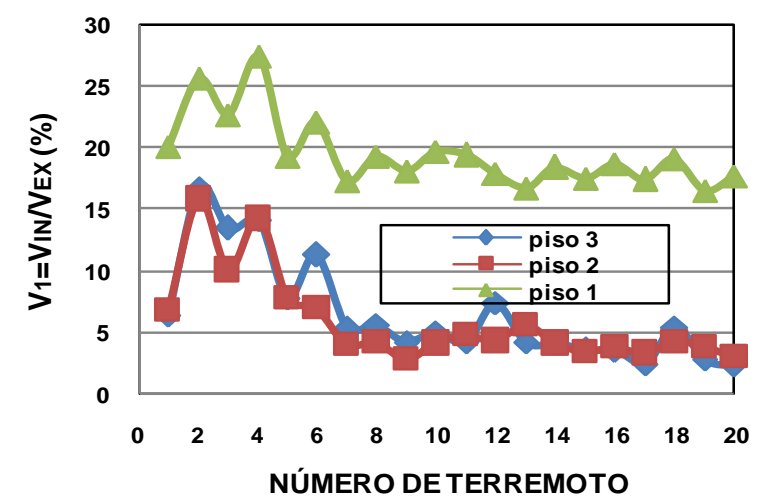

a) Modelo 1

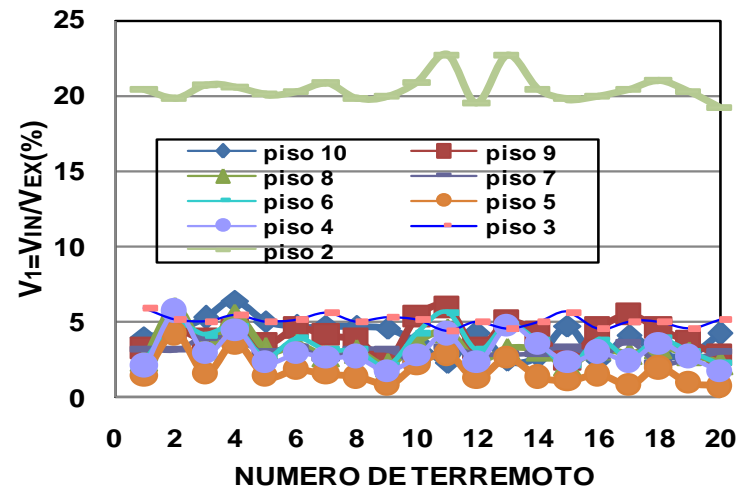

b) Modelo 2

Figura 5. Valores del Parámetro $V_{l}$, dirección $Y$

Los modelos no desarrollaron articulaciones plásticas cuando se excitaron por los 20 sismos. Para estudiar el efecto del comportamiento inelástico en los valores del parámetro $V_{l}$, los sismos se escalaron de tal manera que produjeron fluencia en los modelos. Basado en experiencias anteriores y para una comparación uniforme, los terremotos fueron escalados hasta lograr que el máximo desplazamiento de entrepiso promedio fuera cercano a 1\%. Se observó que se formaron alrededor de 3 a 8 articulaciones plásticas para el desplazamiento de entrepiso deseado. Figuras similares a la figura 5 también se desarrollaron para este caso pero no se muestran. Las estadísticas (media $\mu$, desviación estándar $\sigma$ y coeficiente de variación $\delta$ ) se resumen en la tabla 3 para los dos modelos, direcciones y niveles de deformación. Se observa que la contribución de los MGs a la resistencia lateral puede ser significativa y consecuentemente debe ser considerada en el diseño del sistema estructural en estudio.

Tabla 3. Estadísticas del parámetro $V_{l}(\%)$

\begin{tabular}{|c|c|c|c|c|c|c|c|c|c|c|c|c|c|}
\hline \multirow{3}{*}{ MODELO } & \multirow{3}{*}{ PISO } & \multicolumn{6}{|c|}{ ELÁSTICO } & \multicolumn{6}{|c|}{ INELÁSTICO } \\
\hline & & \multicolumn{3}{|c|}{ DIRECCIÓN X } & \multicolumn{3}{|c|}{ DIRECCIÓN Y } & \multicolumn{3}{|c|}{ DIRECCIÓN X } & \multicolumn{3}{|c|}{ DIRECCIÓN Y } \\
\hline & & $\mu$ & $\sigma$ & $\delta$ & $\mu$ & $\sigma$ & $\delta$ & $\mu$ & $\sigma$ & $\delta$ & $\mu$ & $\sigma$ & $\delta$ \\
\hline \multirow{3}{*}{1} & 1 & 26.2 & 3.7 & 0.14 & 16.0 & 0.7 & 0.04 & 19.5 & 2.9 & 0.15 & 17.6 & 1.9 & 0.11 \\
\hline & 2 & 7.2 & 2.8 & 0.39 & 9.1 & 2.4 & 0.26 & 5.8 & 3.6 & 0.63 & 9.2 & 2.6 & 0.28 \\
\hline & 3 & 7.6 & 3.8 & 0.50 & 7.7 & 3.8 & 0.49 & 6.4 & 4.2 & 0.65 & 7.9 & 3.6 & 0.45 \\
\hline \multirow{9}{*}{2} & 2 & 20.4 & 0.9 & 0.04 & 35.3 & 1.6 & 0.05 & 20.5 & 0.9 & 0.04 & 35.5 & 1.7 & 0.05 \\
\hline & 3 & 5.1 & 0.4 & 0.07 & 6.3 & 0.8 & 0.13 & 5.1 & 0.4 & 0.08 & 6.3 & 0.9 & 0.14 \\
\hline & 4 & 3.0 & 1.1 & 0.37 & 4.4 & 1.6 & 0.36 & 3.0 & 1.0 & 0.35 & 4.4 & 1.5 & 0.35 \\
\hline & 5 & 1.7 & 0.9 & 0.55 & 2.8 & 1.3 & 0.45 & 1.7 & 0.9 & 0.54 & 2.9 & 1.3 & 0.45 \\
\hline & 6 & 3.5 & 1.0 & 0.29 & 5.7 & 2.0 & 0.34 & 3.5 & 1.0 & 0.29 & 5.7 & 2.0 & 0.34 \\
\hline & 7 & 3.0 & 0.4 & 0.12 & 4.9 & 0.8 & 0.15 & 3.1 & 0.5 & 0.15 & 4.9 & 0.8 & 0.15 \\
\hline & 8 & 3.2 & 1.0 & 0.32 & 6.0 & 2.0 & 0.34 & 3.2 & 1.0 & 0.31 & 6.0 & 2.0 & 0.34 \\
\hline & 9 & 3.9 & 0.9 & 0.24 & 7.4 & 2.2 & 0.30 & 4.1 & 1.0 & 0.24 & 7.4 & 2.2 & 0.30 \\
\hline & 10 & 3.9 & 1.1 & 0.28 & 6.6 & 1.3 & 0.20 & 4.0 & 1.1 & 0.28 & 6.6 & 1.3 & 0.19 \\
\hline
\end{tabular}

La magnitud del parámetro $V_{l}$ se estima ahora considerando la rigidez de las conexiones de los MGs. Es importante mencionar que, debido a que no hay información en el reporte (FEMA 355C) en relación a las conexiones de los MGs de los modelos, se diseña una conexión típica a base de ángulos 
dobles en el alma. Después, asumiendo las propiedades de la conexión (espesor, longitud, etc), los parámetros del Modelo de Richard ( $k, M o, K p$ y $N$ ) se obtienen usando el programa PRCONN desarrollado por Richard y su equipo de investigadores (Richard 1993). Los parámetros obtenidos para estas conexiones son $\mathrm{k}=16,400 \mathrm{kN}-\mathrm{m}, \mathrm{kp}=851 \mathrm{kN}-\mathrm{m}, \mathrm{Mo}=72 \mathrm{kN}-\mathrm{m}$ y N=2, para la conexión de la viga de sección W16x26. Los valores correspondientes de estos parámetros para la otra viga (sección W18x35) son 28,800 kN-m, $1448 \mathrm{kN}-\mathrm{m}, 109 \mathrm{kN}-\mathrm{m}$ y 2, respectivamente.

Los resultados de $V_{1}$ se muestran en la figura 6 para la dirección $Y$ y el Modelo 1. Se observa que la contribución de los MGs a la resistencia lateral se incrementa cuando se considera la rigidez de las conexiones. El incremento es particularmente importante para los pisos más altos. Por ejemplo, para el piso $3, \mathrm{~V}_{1}$ resultó menor que $10 \%$ en casi todos los casos con conexiones PA. Para las conexiones SR, sin embargo, este parámetro toma valores mayores a $20 \%$ en casi todos los casos. Valores cercanos a $35 \%$ se observan para el Piso 3 en dos ocasiones. Puesto que las fuerzas generadas por esta contribución no son consideradas en los diseños de los MGs, su capacidad de resistencia puede ser inferior a la que se asume, es decir su diseño puede resultar no conservador

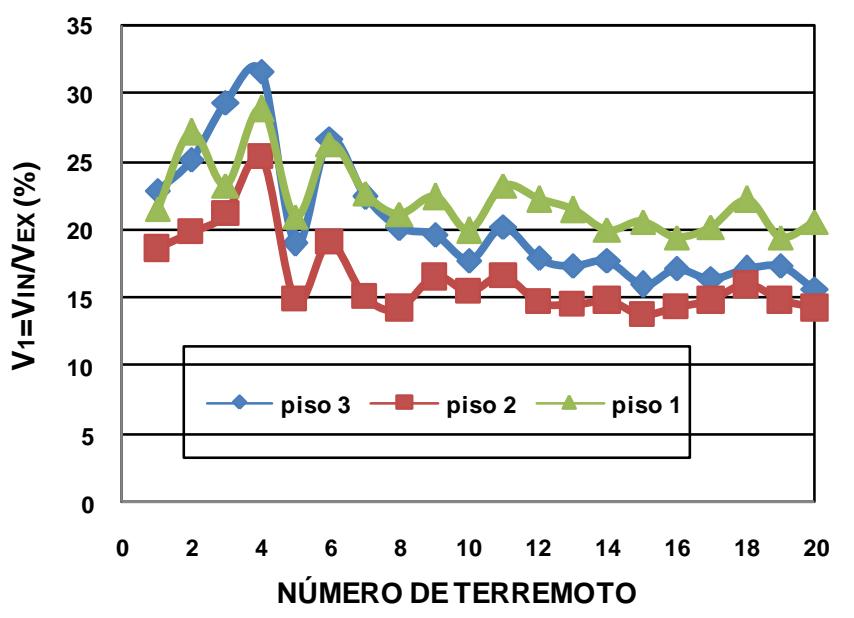

Figura 6. Valores de $V_{1}$, dirección $Y$, Modelo 1, SR

\section{MODELO 3D CON CONEXIONES PA Y MODELO 2D}

En esta sección la respuesta sísmica de los edificios modelados como marcos planos (modelo 2D) se compara con la de los edificios modelados como marcos tridimensionales (modelo 3D) con conexiones PA. Las respuestas se expresan en términos de cortantes de entrepiso, desplazamientos de entrepiso y elementos mecánicos en algunos miembros de la base de los modelos. El cortante de entrepiso se discute primero. La razón de cortantes $V_{2}$, definida como $V_{2 D} / V_{3 D}$, se usa para este propósito. Para una dirección y entrepiso dados, $V_{2 D}$ representará el cortante resistido por todas las columnas del entrepiso en cuestión cuando el edificio se modela como un marco plano mientras que $V_{3 D}$ representará lo mismo pero el cortante ahora se estima para las columnas del marco plano correspondiente del edificio modelado tridimensionalmente. La razón $V_{2}$ se calcula para las dos direcciones horizontales. Como se comento anteriormente, el modelo 3D se excita por ambas componentes $[(X, Y)]$ mientras que el modelo 2D por una componente a la vez, esto es por $(X, O)$ primero y $(Y, O)$ después. 
Los resultados del parámetro $V_{2}$, en porcentaje, se muestran en las figuras 7a y $7 \mathrm{~b}$ para los Modelos 1 y 2 respectivamente, para la dirección $X$. Se observa que los valores de $V_{2}$ varían significativamente de un modelo a otro y de un piso a otro sin mostrar alguna tendencia. En casi todos los casos los valores de $V_{2}$ son mayores que $100 \%$ indicando que, en general, el cortante de entrepiso es más grande para el modelo 2D que para el modelo 3D. Esto se debe a que algunos elementos considerados en el modelo 3D contribuyen a la resistencia y rigidez de la estructura, los que en el modelo $2 \mathrm{D}$ no es posible considerar, así como también a las diferentes características dinámicas y de disipación de energía de las dos modelaciones. En algunos casos se observan valores mayores a $150 \%$. En general, los valores de $V_{2}$ son mayores para el piso inferior. Al igual que para la dirección $X$, también se desarrollaron gráficas para la dirección $Y$ pero no se muestran. En general las observaciones anteriores son validas para esta dirección.

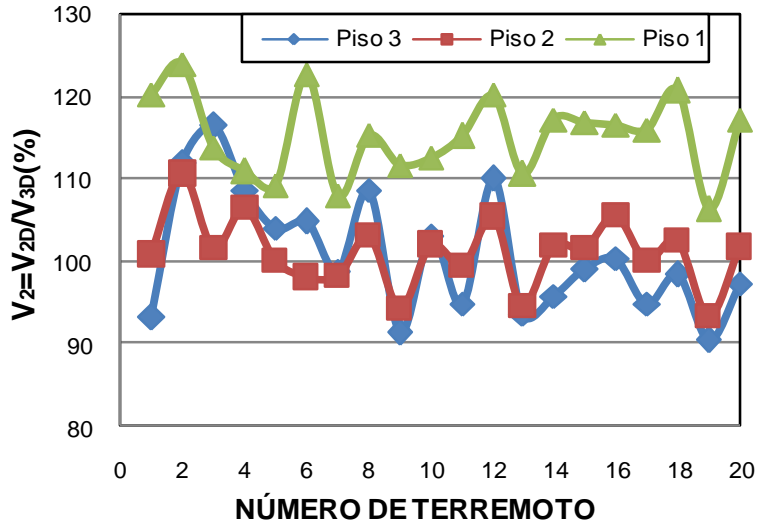

a) Modelo 1

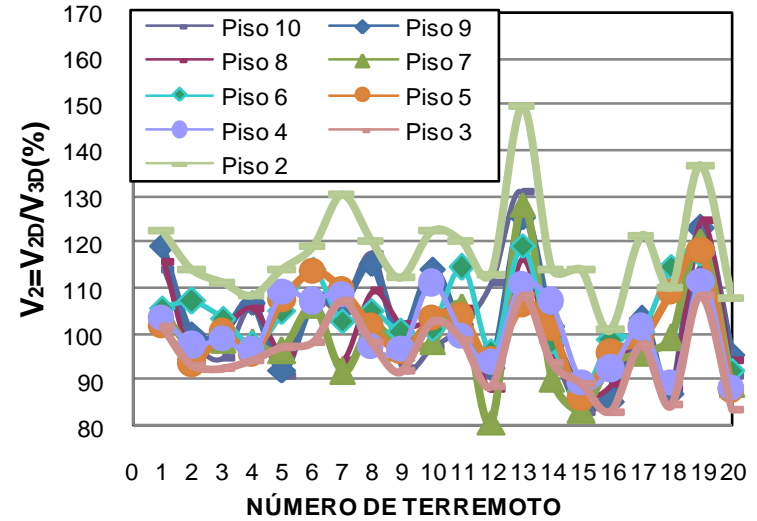

b) Modelo 2

Figura 7. Valores del Parámetro $V_{2}$, dirección $X$

Un parámetro similar al del cortante de entrepiso $\left(V_{2}\right)$ se estima para los desplazamientos de entrepiso $\left(D_{1}\right)$. Los resultados, para la dirección $X$, se dan en la figura 8. Los resultados indican que, como en la discusión de $V_{2}$, los valores de $D_{1}$ son mayores que $100 \%$ en la mayoría de los casos. Una razón similar a las anteriores también se estima para carga axial $\left(A_{l}\right)$ y momento $\left(M_{l}\right)$ para algunas columnas de la base. Columnas interiores y exteriores (mostradas en la figura 4) de los MRMs se consideran. Los resultados para el Modelo 1 se muestran en la figura 9. Las observaciones hechas para $V_{2}$ y $D_{1}$ son en general también válidas para estos dos parámetros. Los resultados muestran que los valores más grandes de $A_{1}$ y $M_{1}$ ocurren para las columnas interiores, valores cercanos a $160 \%$ se observan en algunos casos. Los valores para las dos columnas exteriores son esencialmente los mismos. Con base en estos resultados, se concluye que, la modelación de los edificios considerándolos como marcos planos, puede resultar en una sobreestimación significativa en la respuesta sísmica. Figuras similares a las 7, 8 y 9 también se desarrollan para el caso inelástico para los dos modelos y las dos direcciones pero no se muestran. Se observa, sin embargo, que como la fluencia no es muy significativa los valores son muy similares para comportamiento elástico e inelástico. Las estadísticas de estos parámetros se dan en las Tablas 4, 5, y 6. De dichas tablas se observa que, en general, los valores de $V_{2}$ y $D_{2}$ son mayores para el Modelo 2 que para el Modelo 1. El nivel de incertidumbre en la estimación de estos parámetros es similar para los dos modelos. 


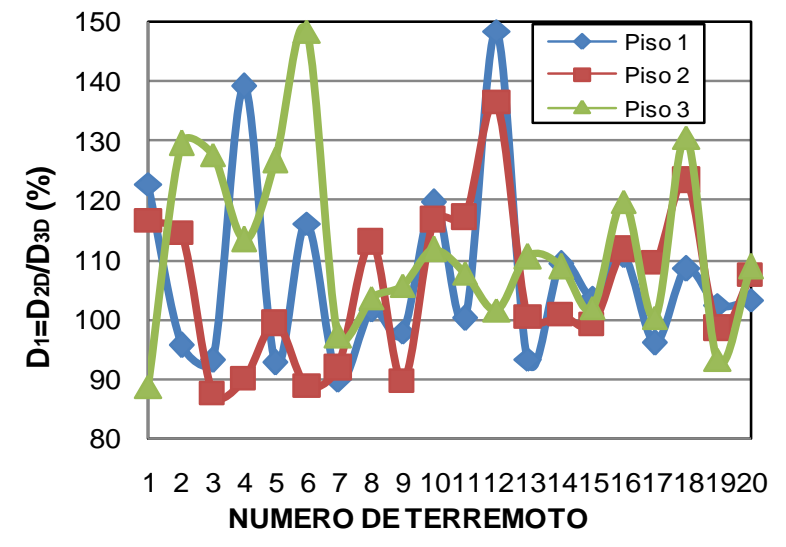

a) Modelo 1

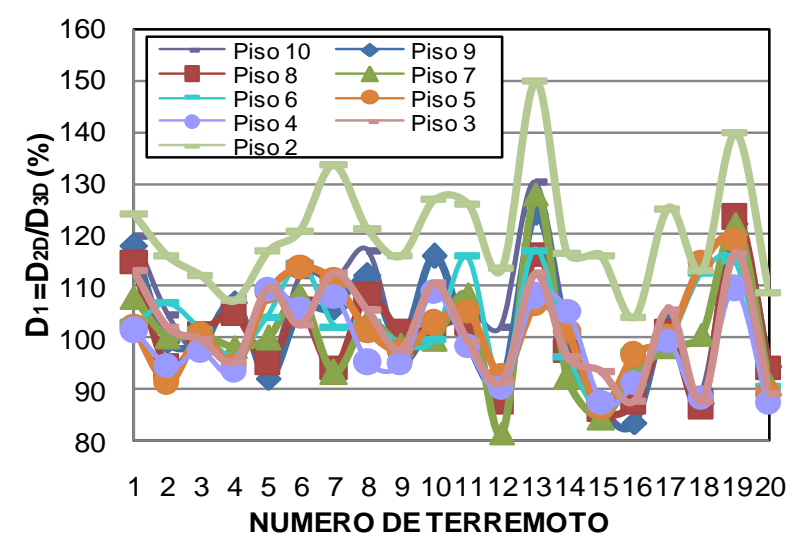

b) Modelo 2

Figura 8. Valores del Parámetro $D_{l}$, dirección $X$

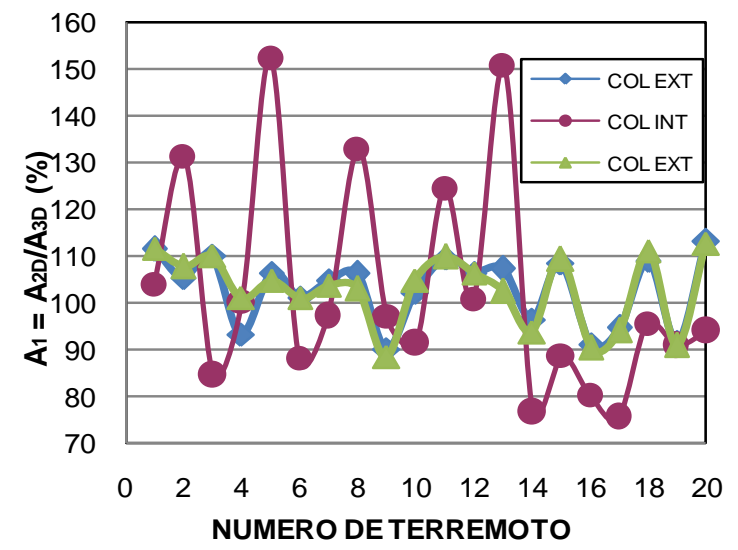

a) Razón de cargas axiales

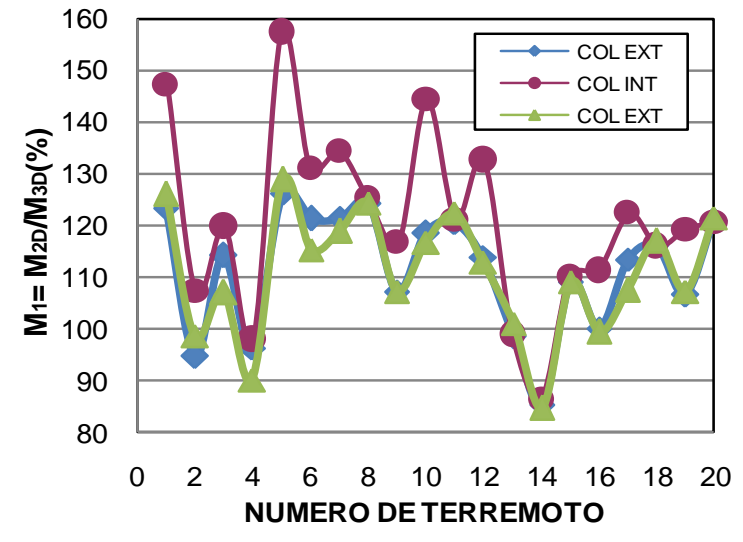

b) Razón de momentos

Figura 9. Valores de los parámetros $A_{l}$ y $M_{l}$, Modelo 1

\section{MODELO 3D CON CONEXIONES PA Y SR}

El efecto de la rigidez de la conexión en la respuesta estructural, en términos de cortantes y desplazamientos de entrepiso y elementos mecánicos se discute en esta parte del artículo. Únicamente el Modelo 1 y la dirección $Y$ se consideran. Los resultados se presentan tanto para los promedios de todos los marcos como para marcos individuales.

\section{Promedios de todos los marcos}

El cortante de entrepiso se discute primero. El parámetro $V_{3}$, definido como $V_{P A} / V_{S R}$ se usa para este fin. Para un piso dado, $V_{P A}$ representará el cortante promedio de todos los marcos en ese piso cuando las conexiones se consideran como PA en los MGs del modelo tridimensional. $V_{S R}$ representará lo mismo, excepto que las conexiones se asumen SR. 
Tabla 4. Estadísticas del parámetro $V_{2}$

\begin{tabular}{|c|c|c|c|c|c|c|c|c|c|c|c|c|c|}
\hline \multirow{3}{*}{ MODELO } & \multirow{3}{*}{ PISO } & \multicolumn{6}{|c|}{ ELÁSTICO } & \multicolumn{6}{|c|}{ INELÁSTICO } \\
\hline & & \multicolumn{3}{|c|}{ DIRECCIÓN X } & \multicolumn{3}{|c|}{ DIRECCIÓN Y } & \multicolumn{3}{|c|}{ DIRECCIÓN X } & \multicolumn{3}{|c|}{ DIRECCIÓN Y } \\
\hline & & $\mu$ & $\sigma$ & $\delta$ & $\mu$ & $\sigma$ & $\delta$ & $\mu$ & $\sigma$ & $\delta$ & $\mu$ & $\sigma$ & $\delta$ \\
\hline \multirow{6}{*}{1} & 1 & 115.2 & 4.9 & 0.04 & 97.6 & 11.9 & 0.12 & 114.5 & 5.1 & 0.04 & 103.4 & 11.4 & 0.11 \\
\hline & 2 & 101.0 & 4.2 & 0.04 & 101.6 & 9.8 & 0.10 & 100.6 & 4.3 & 0.04 & 105.0 & 7.8 & 0.07 \\
\hline & 3 & 100.7 & 7.4 & 0.07 & 96.9 & 12.0 & 0.12 & 100.3 & 7.1 & 0.07 & 98.1 & 11.0 & 0.11 \\
\hline & 2 & 118.2 & 10.9 & 0.09 & 140.3 & 13.2 & 0.09 & 116.5 & 8.7 & 0.07 & 137.9 & 9.3 & 0.07 \\
\hline & 3 & 95.8 & 7.7 & 0.08 & 101.2 & 5.9 & 0.06 & 94.6 & 6.0 & 0.06 & 99.8 & 5.3 & 0.05 \\
\hline & 4 & 100.5 & 7.8 & 0.08 & 106.4 & 7.8 & 0.07 & 99.5 & 6.4 & 0.06 & 105.3 & 7.0 & 0.07 \\
\hline \multirow{6}{*}{2} & 5 & 101.3 & 8.2 & 0.08 & 104.3 & 6.5 & 0.06 & 100.2 & 8.0 & 0.08 & 103.0 & 5.2 & 0.05 \\
\hline & 6 & 104.2 & 8.5 & 0.08 & 107.8 & 7.1 & 0.07 & 103.2 & 8.7 & 0.08 & 106.5 & 6.9 & 0.06 \\
\hline & 7 & 98.9 & 11.1 & 0.11 & 101.1 & 6.4 & 0.06 & 97.7 & 10.4 & 0.11 & 99.9 & 6.2 & 0.06 \\
\hline & 8 & 101.1 & 10.3 & 0.10 & 107.3 & 7.8 & 0.07 & 99.8 & 8.7 & 0.09 & 106.0 & 7.5 & 0.07 \\
\hline & 9 & 103.2 & 11.8 & 0.11 & 109.7 & 9.9 & 0.09 & 101.5 & 9.8 & 0.10 & 108.6 & 9.6 & 0.09 \\
\hline & 10 & 101.8 & 12.0 & 0.12 & 101.8 & 7.9 & 0.08 & 100.0 & 9.8 & 0.10 & 100.7 & 7.5 & 0.07 \\
\hline
\end{tabular}

Tabla 5. Estadísticas del parámetro $D_{l}(\%)$

\begin{tabular}{|c|c|c|c|c|c|c|c|c|c|c|c|c|c|}
\hline \multirow{3}{*}{ MODELO } & \multirow{3}{*}{ PISO } & \multicolumn{6}{|c|}{ ELÁSTICO } & \multicolumn{6}{|c|}{ INELÁSTICO } \\
\hline & & \multicolumn{3}{|c|}{ DIRECCIÓN X } & \multicolumn{3}{|c|}{ DIRECCIÓN Y } & \multicolumn{3}{|c|}{ DIRECCIÓN X } & \multicolumn{3}{|c|}{ DIRECCIÓN Y } \\
\hline & & $\mu$ & $\sigma$ & $\delta$ & $\mu$ & $\sigma$ & $\delta$ & $\mu$ & $\sigma$ & $\delta$ & $\mu$ & $\sigma$ & $\delta$ \\
\hline \multirow{6}{*}{1} & 1 & 111.8 & 14.8 & 0.13 & 104.5 & 18.4 & 0.18 & 110.6 & 6.6 & 0.06 & 104.0 & 13.4 & 0.13 \\
\hline & 2 & 105.5 & 13.3 & 0.13 & 97.3 & 17.5 & 0.18 & 101.8 & 5.2 & 0.05 & 102.2 & 9.8 & 0.10 \\
\hline & 3 & 107.1 & 15.6 & 0.15 & 89.1 & 19.1 & 0.21 & 102.0 & 7.1 & 0.07 & 97.9 & 12.9 & 0.13 \\
\hline & 2 & 120.4 & 11.1 & 0.09 & 123.9 & 10.8 & 0.09 & 119.2 & 9.5 & 0.08 & 122.9 & 9.6 & 0.08 \\
\hline & 3 & 101.5 & 9.0 & 0.09 & 108.7 & 6.4 & 0.06 & 101.0 & 8.2 & 0.08 & 109.0 & 7.9 & 0.07 \\
\hline & 4 & 98.8 & 7.8 & 0.08 & 100.6 & 7.8 & 0.08 & 97.6 & 7.2 & 0.07 & 100.3 & 7.6 & 0.08 \\
\hline \multirow{6}{*}{2} & 5 & 101.8 & 8.7 & 0.09 & 102.2 & 6.2 & 0.06 & 100.5 & 9.4 & 0.09 & 101.1 & 5.7 & 0.06 \\
\hline & 6 & 102.7 & 8.8 & 0.09 & 100.5 & 6.6 & 0.07 & 101.4 & 9.2 & 0.09 & 99.0 & 6.7 & 0.07 \\
\hline & 7 & 100.6 & 11.0 & 0.11 & 100.8 & 6.5 & 0.06 & 99.3 & 10.3 & 0.10 & 99.5 & 6.2 & 0.06 \\
\hline & 8 & 100.2 & 10.2 & 0.10 & 101.8 & 7.3 & 0.07 & 99.2 & 9.3 & 0.09 & 100.7 & 6.9 & 0.07 \\
\hline & 9 & 102.4 & 11.6 & 0.11 & 103.4 & 9.3 & 0.09 & 101.2 & 10.6 & 0.10 & 102.4 & 9.4 & 0.09 \\
\hline & 10 & 104.2 & 11.7 & 0.11 & 107.2 & 8.4 & 0.08 & 102.5 & 9.9 & 0.10 & 106.1 & 8.3 & 0.08 \\
\hline
\end{tabular}

Tabla 6. Estadísticas de los parámetros $A_{l}$ y $M_{l}(\%)$

\begin{tabular}{|c|c|c|c|c|c|c|c|c|c|c|c|c|c|c|}
\hline \multirow{3}{*}{ PARAMETRO } & \multirow{3}{*}{ MODELO } & \multirow{3}{*}{ ELEM. } & \multicolumn{6}{|c|}{ ELÁSTICO } & \multicolumn{6}{|c|}{ INELÁSTICO } \\
\hline & & & \multicolumn{3}{|c|}{ DIRECCIÓN X } & \multicolumn{3}{|c|}{ DIRECCIÓN Y } & \multicolumn{3}{|c|}{ DIRECCIÓN X } & \multicolumn{3}{|c|}{ DIRECCIÓN Y } \\
\hline & & & $\mu$ & $\sigma$ & $\delta$ & $\mu$ & $\sigma$ & $\delta$ & $\mu$ & $\sigma$ & $\delta$ & $\mu$ & $\sigma$ & $\delta$ \\
\hline \multirow{6}{*}{ CARGA AXIAL } & \multirow{3}{*}{1} & EXT & 103 & 4.1 & 0.04 & 101 & 9.4 & 0.09 & 102 & 3.5 & 0.03 & 103 & 7.4 & 0.07 \\
\hline & & INT & 114 & 5.3 & 0.05 & 95 & 17.7 & 0.19 & 132 & 30.8 & 0.23 & 103 & 23.1 & 0.22 \\
\hline & & EXT & 101 & 4.1 & 0.04 & 101 & 9.7 & 0.10 & 100 & 3.5 & 0.03 & 103 & 7.6 & 0.07 \\
\hline & 2 & INT & 101 & 8.8 & 0.09 & 109 & 22.2 & 0.20 & 100 & 8.8 & 0.09 & 134 & 42.1 & 0.31 \\
\hline & & EXT & 114 & 5.4 & 0.05 & 106 & 12.2 & 0.11 & 113 & 5.6 & 0.05 & 111 & 11.5 & 0.10 \\
\hline & & INT & 114 & 5.0 & 0.04 & 106 & 12.2 & 0.11 & 114 & 5.3 & 0.05 & 121 & 17.3 & 0.14 \\
\hline \multirow[t]{2}{*}{ MOMENTO } & & EXT & 113 & 4.2 & 0.04 & 105 & 12.7 & 0.12 & 112 & 4.3 & 0.04 & 111 & 11.9 & 0.11 \\
\hline & 2 & INT & 114 & 10.3 & 0.09 & 117 & 10.4 & 0.09 & 112 & 8.7 & 0.08 & 117 & 9.3 & 0.08 \\
\hline
\end{tabular}

Los resultados de $V_{3}$ se muestran en la figura 10a. Se observa que los valores son menores que $100 \%$ en la mayoría de los casos, indicando que el cortante de entrepiso se incrementa cuando se 
considera la rigidez de la conexión. Sin embargo, para otros casos, $V_{3}$ es mayor que $100 \%$ indicando que el cortante de entrepiso decrece cuando las conexiones son consideradas. A diferencia de lo que sucede en el caso de aplicación de carga estática lateral, donde el cortante de entrepiso se espera que siempre aumente con la rigidez de la conexión, la respuesta debida a carga dinámica depende de varios parámetros los cuales son menos significativos para análisis estático. Entre ellos podemos mencionar la distribución de masa y rigidez, disipación de energía, distribución de deformaciones inelásticas en la estructura, efectos de los modos de vibrar y contenido de frecuencias de los sismos. Ha sido mostrado en otras investigaciones para marcos planos (Reyes-Salazar y Haldar2001a, Reyes-Salazar y Haldar2001b) que si se incrementa la rigidez de las conexiones, el cortante de entrepiso se incrementa pero no para todos los casos. También ha sido mostrado (Reyes-Salazar y Haldar2001b) que la disipación de energía en conexiones SR puede ser comparable, e incluso más grande, que la disipación de energía por amortiguamiento viscoso y comportamiento histerético en articulaciones plásticas. El efecto de los modos superiores de vibrar, disipación de energía y contenido de frecuencias de los sismos es claramente ilustrado en la figura 10a; los valores de $V_{3}$ significativamente varían de un sismo a otro aun cuando la deformación máxima relativa de entrepiso es aproximadamente la misma para todos los sismos $(\approx 1 \%)$. La implicación de ésto es que el comportamiento sísmico de un edificio con conexiones SR puede ser muy diferente a la del modelo idealizado con conexiones PA.

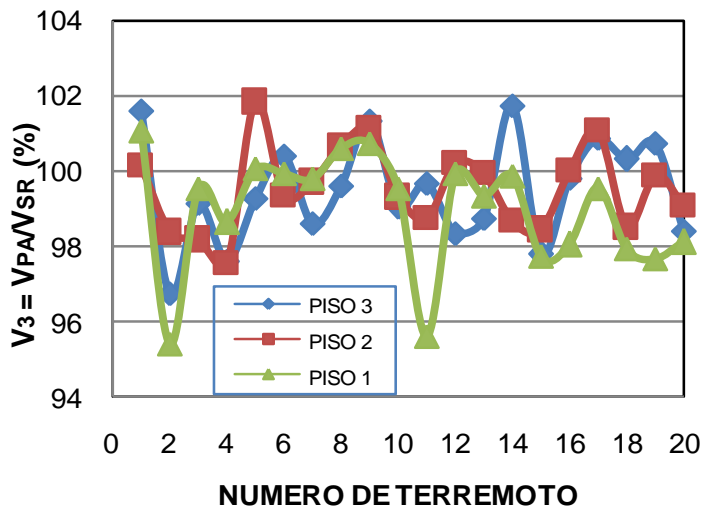

a) Cortantes promedio

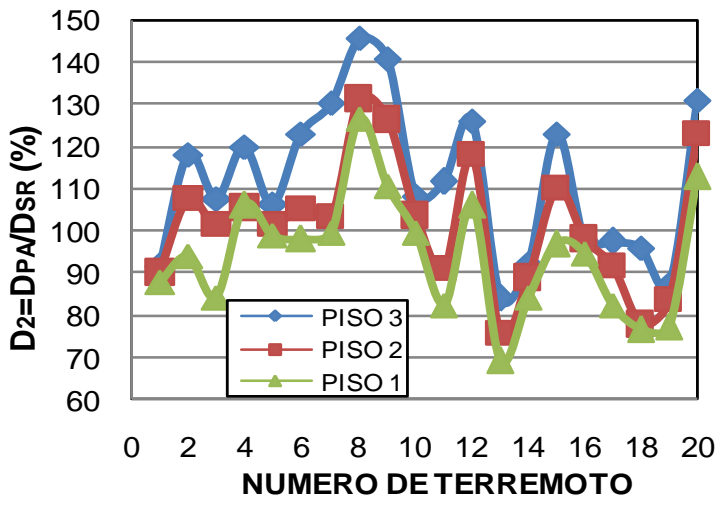

b) Desplazamientos promedio

Figura 10. Valores de los parámetros $V_{3}$ y $D_{2}$, dirección $X$

Los resultados para desplazamientos de entrepiso se discuten a continuación. El parámetro $D_{2}$, definido como $D_{P A} / D_{S R}$ se usa en este caso. Las notaciones $D_{P A}$ y $D_{S R}$ representan el promedio de desplazamiento de entrepiso para los marcos con conexiones PA y SR, respectivamente. Los valores de $D_{2}$ se muestran en la figura $10 \mathrm{~b}$. Al igual que para el parámetro $V_{3}$, se observa que los valores de $D_{2}$ varían de un sismo a otro y de un piso a otro sin mostrar tendencia alguna. Se observan valores mayores que $100 \%$ en la mayoría de los casos, particularmente para el piso 3. Esto implica que los desplazamientos promedios de los marcos con conexiones PA son en general mayores que los obtenidos en los marcos con conexiones SR.

Resultados en términos de carga axial y momento en las columnas de la base antes mencionadas también se estiman. Los parámetros $A_{2}$ y $M_{2}$, para carga axial y momento, se usan para ese propósito. Los resultados se muestran en la figura 11. Se observa que los valores de estos parámetros en algunos casos se incrementan, y en algunos otros disminuyen, cuando se considera la rigidez de las conexiones. 


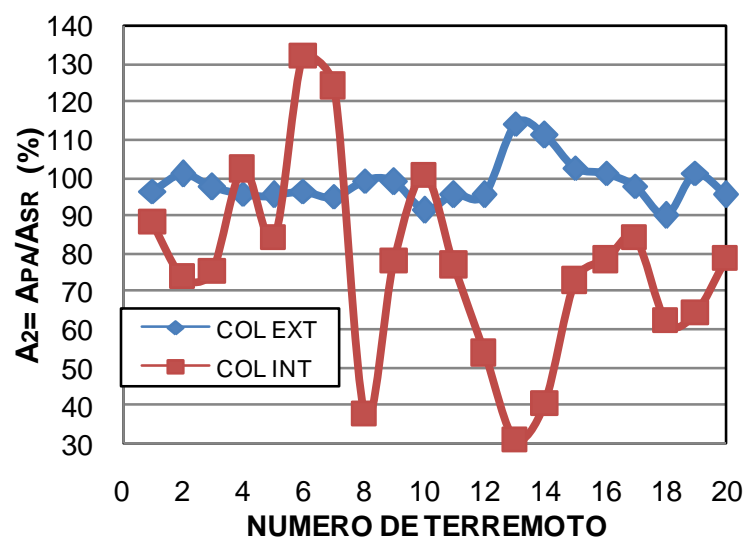

a) Razón de cargas axiales

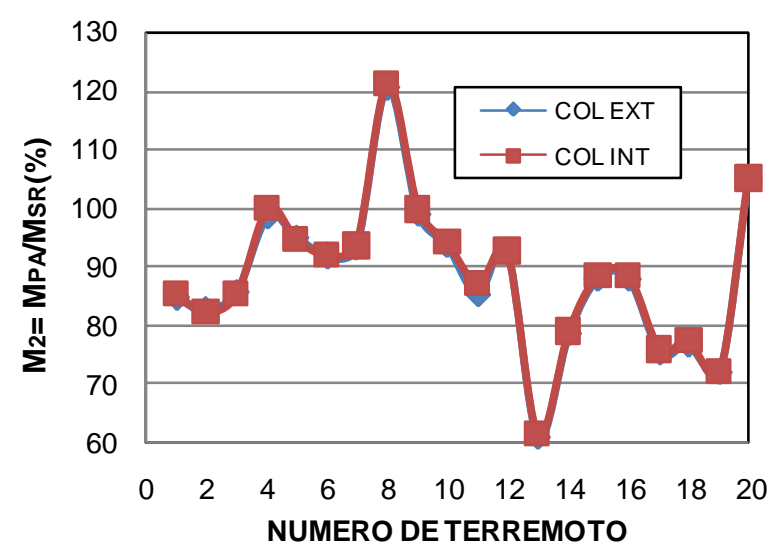

b) Razón de momentos

Figura 11. Valores de los parámetros $A_{2}$ y $M_{2}$, dirección $Y$

\section{Marcos Individuales}

La razón de cortantes de entrepiso del Modelo 1 con conexiones PA y del Modelo 1 con conexiones SR se discute a continuación para marcos individuales. Se presentan los resultados únicamente para la dirección $Y$ que es la dirección de orientación de las conexiones SR. Para la otra dirección la razón de cortantes es cercana a $100 \%$. El mismo parámetro $V_{3}$, usado para el cortante promedio, se usa para el caso de cortantes en marcos individuales. Los resultados se muestran en la figura12apara marcos exteriores (MRM). En este caso, para un piso dado, $V_{P A}$ representará la aportación a la resistencia lateral de ese piso para el modelo con conexiones PA. $V_{S R}$ representará lo mismo, pero para el modelo con conexiones SR. Los resultados de la figura 12a indican que los cortantes de entrepiso del marco exterior son en general mayores para el modelo con conexiones PA que para el modelo con conexiones SR. Esto es coherente; la contribución a la resistencia lateral de los MGs del modelo con conexiones PA es pequeña pues proviene únicamente de las columnas exteriores que son parte de los MRMs transversales ubicados en el plano $X-Z$ (ver figura 3), por lo tanto la resistencia lateral $\left(V_{P A}\right)$ es mayormente proporcionada por los marcos exteriores. Por el contrario, la aportación de los MGs a la resistencia lateral significativamente se incrementa cuando se consideran conexiones SR y en consecuencia decrece la aportación a la resistencia $\left(V_{S R}\right)$ de los marcos exteriores.

Los valores de $V_{3}$ para los marcos interiores se muestran en la figura $12 \mathrm{~b}$. El significado de $V_{P A} \mathrm{y}$ $V_{S R}$ es el mismo que para MRMs pero ahora se trata de cortantes de entrepiso en los MGs. Como era de esperarse, los valores de $V_{3}$ resultan menores que $100 \%$ prácticamente en todos los casos puesto que, como se comentó anteriormente para marcos interiores, su aportación a la resistencia lateral $\left(V_{P A}\right)$ es pequeña. Dicha aportación $\left(V_{S R}\right)$ se incrementa significativamente cuando se consideran conexiones SR.

Los resultados en términos de desplazamientos individuales se presentan en las figuras $13 \mathrm{a}$ y $13 \mathrm{~b}$ para marcos exteriores interiores, respectivamente. Se observa que las magnitudes de dichos desplazamientos son prácticamente iguales para marcos exteriores y exteriores, los que a su vez son muy similares a los desplazamiento promedio discutido anteriormente (figura 10b). Esto es de esperarse; como se comentó en la introducción del artículo; el efecto de diafragma rígido introducido por la rigidez de la losa hace que la deformación lateral en cada uno de los marcos sea esencialmente la misma. 


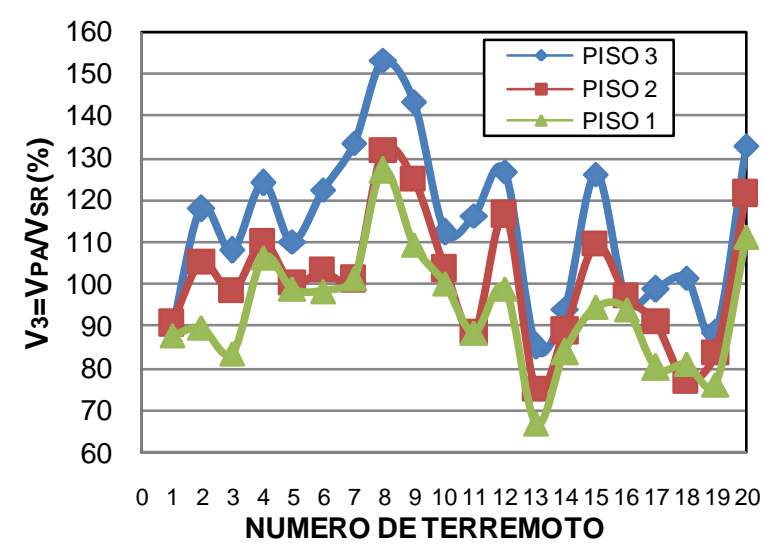

a) Marco exterior

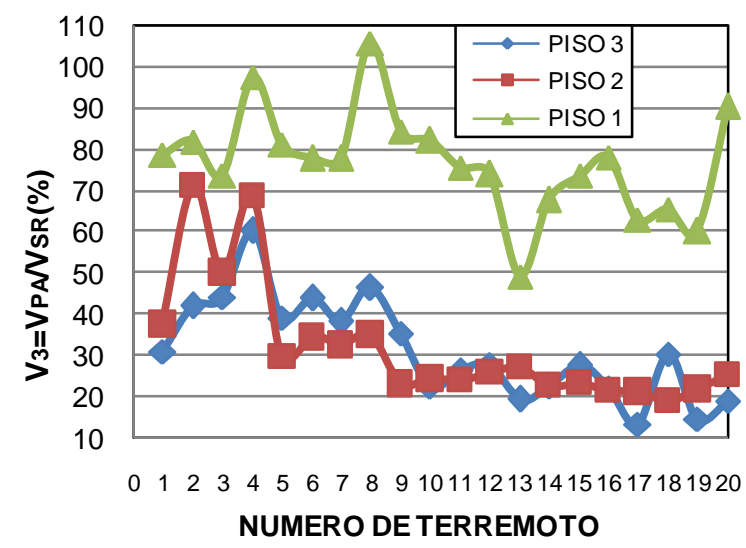

b) Marco interior

Figura 12. Valores del parámetro $V_{3}$ para marcos individuales

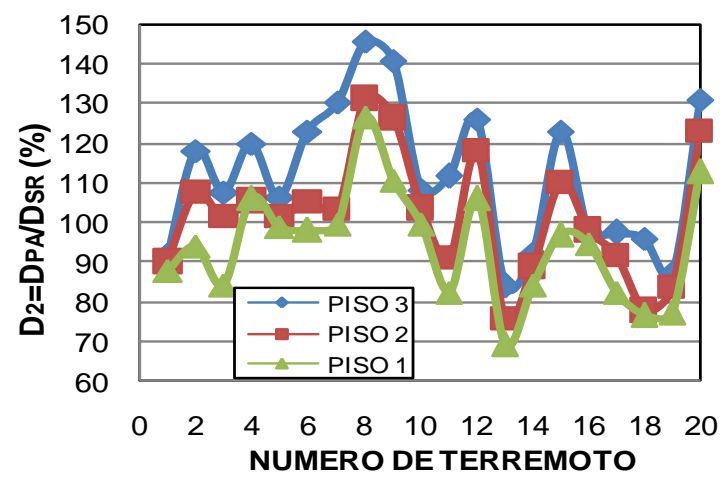

a) Marco exterior

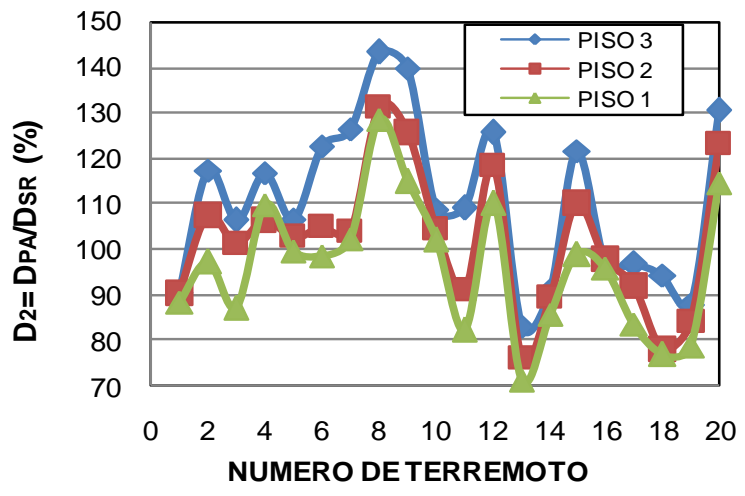

b) Marco interior

Figura 13. Valores del parámetro $D_{2}$ para marcos individuales

\section{MAGNITUD DE LOS MOMENTOS EN LAS CONEXIONES}

Como se comentó en secciones anteriores, para fines de simplificación, se asume que la conexión viga columna de los MGs es PA. En esta parte del artículo se estudia la importancia relativa del momento máximo desarrollado en las conexiones en relación al momento plástico de las vigas que conectan. El parámetro $M_{3}$, definido como $M_{C O N} / M_{P}$ se usa para este propósito. $M_{C O N}$ representa el momento máximo desarrollado en la conexión y $M_{P}$ el momento plástico de la viga. El parámetro $M_{3}$ se calcula para las primeras tres vigas del MG central orientadas en dirección $Y$, una en cada nivel y se presentan en la figura 14. Se observan valores desde un 10 hasta un 30\%, lo que implica que la magnitud de los momentos que las conexiones transmiten puede ser considerable. Los resultados mostrados corresponden al caso de una deformación correspondiente a fluencia moderada en donde el desplazamiento de entrepiso es del orden del 1\%. Para deformaciones mayores se espera que el parámetro $M_{3}$ alcance valores más grandes. Lo anterior valida las conclusiones de las secciones anteriores: la contribución de la conexión a la rigidez y disipación de energía de las estructuras es muy importante y no debe ser ignorada en el análisis sísmico del sistema estructural en consideración. 


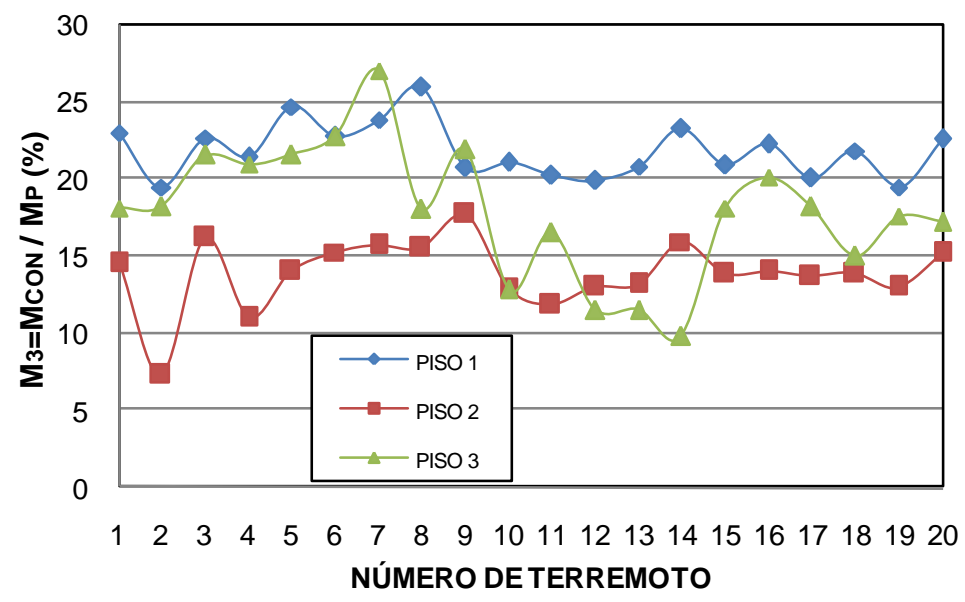

Figura 14. Valores del parámetro $M_{3}$

\section{CONCLUSIONES}

Los resultados de esta investigación indican que la contribución de los MGs a la resistencia lateral puede ser significativa. Dicha contribución es más grande en los pisos inferiores para estructuras con conexiones PA y se incrementa cuando se considera la rigidez de la conexión de los MGs, particularmente para pisos superiores. Puesto que las fuerzas generadas por esta contribución no se consideran en el diseño de los MGs, su capacidad de resistencia puede ser inferior a la que se asume, en otras palabras su capacidad puede ser sobrestimada. Se muestra que modelar los edificios como marcos planos puede resultar en elementos mecánicos, cortantes y desplazamientos de entrepiso más grandes que cuando se modelan en forma tridimensional, por lo que su diseño puede ser conservador. También se observa que el cortante de entrepiso promedio generalmente se incrementa cuando se considera la rigidez de las conexiones. Sin embargo, para algunos casos, el cortante disminuye. Los desplazamientos de entrepiso promedio son similares para los modelos con conexiones PA y SR. Los resultados también indican que los momentos que las conexiones pueden transmitir son hasta del $30 \%$ de los momentos plásticos de las vigas a las que conectan. La diferencia entre los resultados de cada representación estructural se debe principalmente a los elementos que contribuyen a la resistencia y rigidez, y a las características dinámicas diferentes de cada representación estructural. Se concluye que, si se usa la estructuración antes mencionada, el modelo tridimensional debe ser usado en el análisis sísmico del edificio, que los MGs deberán considerarse como parte del sistema lateral resistente y que la rigidez de las conexiones deberá incluirse en el diseño de los MGs.

Es importante comentar que el tipo de estructuración estudiada no es común en México y que aunque los resultados obtenidos se basan en los modelos estructurales particulares descritos en la Sección 5 , se espera que éstos sean cualitativamente iguales para otros modelos de edificios con marcos resistentes a momento perimetrales y marcos de gravedad en el interior, y para otros terremotos con periodos predominantes similares a los considerados en esta investigación. 


\section{AGRADECIMIENTOS}

Esta publicación fue posible gracias al apoyo recibido del Consejo Nacional de Ciencia y Tecnología (CONACyT) a través del proyecto 50298-J y de la Universidad Autónoma de Sinaloa a través del proyecto PROFAPI 2008/153. Las opiniones, conclusiones, o recomendaciones expresadas en esta publicación son de los autores y no necesariamente reflejan la opinión del patrocinador.

\section{REFERENCIAS}

BOCA (1993), “12th Edition Building Officials \& Code Administration International Inc”, National Building Code.

Colson, A (1991), "Theoretical modeling of semi-rigid connections behavior", Journal of Constructional Steel Research, Vol. 19, pp. 13-224.

El-Salti, M K (1992), "Design of frames with partially restrained connections", PhD Thesis, Department of Civil Engineering and Engineering Mechanics, University of Arizona

FEMA (2000), "State of the Art Report on Systems Performance of Steel Moment Frames Subjected to Earthquake Ground Shaking", SAC Steel Project, Report FEMA 355C. Federal Emergency Management Agency

Gao, L and A Haldar (1995), "Nonlinear seismic response of space structures with PR connections", International Journal of Microcomputers in Civil Engineering, Vol. 10, pp. 27-37.

Liu, J, and A Astaneh-Asl (2000), "Cyclic tests on Simple Connections Including Effects of The Slab", Report SAC/BD-00/03, SAC Joint Venture.

Reyes-Salazar, A (1997), "Inelastic Seismic Response and Ductility Evaluation of Steel Frames with Fully, Partially Restrained and Composite Connections", PhD Thesis, Department of Civil Engineering and Engineering Mechanics, University of Arizona.

Reyes-Salazar A and A Haldar, A (1999), "Nonlinear seismic response of steel structures with semi-rigid and composite connections", Journal of Constructional Steel Research, Vol. 51, pp. 37-59.

Reyes-Salazar A and A Haldar (2000), "Dissipation of energy in steel frames with PR connections", Structural Engineering and Mechanics, An International Journal, Vol. 9, No. 3, pp. 241-256.

Reyes-Salazar A and A Haldar (2001a), "Energy dissipation at PR frames under seismic loading", Journal of Structural Engineering ASCE, Vol. 127, No. 5, pp. 588-593.

Reyes-Salazar A and A Haldar (20001b), "Seismic response and energy dissipation in partially restrained and fully restrained steel frames: an analytical study", Steel \& Composite Structures, An International Journal, Vol. 1, No. 4, pp. 459-480.

Reyes Salazar A, J I Velázquez Dimas J y A López Barraza (2001), "Respuesta sísmica inelástica de marcos de acero resistentes a momento con conexiones rígidas y semi-rígidas", Revista de Ingeniería Sísmica, Vol. 64, pp. 45-68.

Richard RM (1993), "Moment-rotation curves for partially restrained connections", PRCONN, RMR Design Group, Tucson, Arizona.

UBC (1994), "Structural Engineering Design Provisions, Uniform Building Code, Vol. 2", International Conference of Building Officials. 